\title{
FORMACIÓN DE UN CORREDOR DE HÁBITAT DE UN BOSQUE MONTANO ALTO EN UN MOSAICO DE PÁRAMO EN EL NORTE DEL ECUADOR
}

\section{CREATING A HIGH ANDEAN MONTANE FOREST HABITAT CORRIDOR WITHIN A PARAMO MOSAIC IN NORTHERN ECUADOR}

\author{
David Suárez Duque ${ }^{1}$
}

\section{Resumen}

La estación Los Encinos se encuentra en la zona de amortiguamiento de la Reserva Ecológica El Ángel en la provincia del Carchi, en el norte del Ecuador. Aquí el paisaje esta dominado por páramo de frailejones con islas de bosque siempre verde montano alto. Para mantener la funcionalidad de estas islas de bosque se probaron tres estrategias de plantación de especies nativas para formar un corredor de hábitat, de estas se encontró que las especies plantadas en el borde de los remanentes del bosque, se desarrollaron mejor que las puestas alejadas del remanente. Para recuperar los bosques alto andinos es más efectivo utilizar a los remanentes como núcleo para ampliar el área de bosque.

Palabras calve: restauración, corredor, bosques andinos, fragmentación, borde de bosque

\begin{abstract}
The Los Encinos research station is located near El Angel Ecological Reserve in Carchi province, northern Ecuador. The landscape is dominated by frailejón páramo with islands of Andean montane forest. In order to maintain the ecological functionality of the "forest islands", three planting strategies were tested with native species to form a habitat corridor. Species planted on the edge of the forest develop better than those in other sites. This demonstrates that in order to recuperate the native forests, it is more effective to use the remnants as a nucleus to increase the size of the forest.
\end{abstract}

Key words: restoration, corridor, Andean forest, fragmentation, forest, edge

\section{Introducción}

El bosque siempreverde montano alto se extiende en el Ecuador desde los 3000 hasta los $3400 \mathrm{msnm}$, es la vegetación de transición entre el bosque de neblina y el páramo. Este bosque también llamado "Ceja Andina" es muy similar al bosque nublado en su fisonomía y en la cantidad de musgos y plantas epifitas (Valencia et al., 1999) pero difiere en la estructura y tamaño. Actualmente el bosque de Ceja Andina está presente en forma de islas de bosque natural (fragmentos o parches) relegados a las quebradas, o en suelos con pendientes pronunciadas (Luteyn, 1999). Este aislamiento del bosque se debe a varios factores, como los provocados por deslaves, derrumbes u otros desastres naturales (Neill, 1999) y los ocasionados por el ser humano (incendios $\mathrm{y}$ transformación a suelos agrícolas). Esta situación pone en riesgo la sobrevivencia de estos bosques y de la biodiversidad ligada a ellos.

En el norte del Ecuador, en la zona de amortiguamiento de la Reserva Ecológica El Ángel, el bosque de Ceja Andina se encuentra en forma de islas dentro de un matriz de páramo de frailejones (Espeletia pycnophyla Cuatrec). Muchos de estos fragmentos son muy pequeños y por ende se los puede considerar como partes no funcionales de un ecosistema y en peligro de desaparecer. Una de las estrategias que sugiere la ecología de paisaje para mantener la viabilidad de los ecosistemas es la formación de corredores (Bennet, 2003), los cuales se clasifican por su tamaño y objetivos en: corredores biológicos, corredores de conservación, de hábitat, de paisaje entre otros. Los corredores de hábitat pretenden unir fragmentos del mismo hábitat a escala local (menores a $1 \mathrm{~km}$ ). En este artículo se pone a consideración la estrategia utilizada para la formación de un corredor de hábitat, entre dos fragmentos de bosque de diferente tamaño separados hace más de 40 años.

\section{Materiales y métodos}

Área de estudio

La presente investigación fue desarrollada en la Estación Científica Los Encinos, ubicada al norte el Ecuador en la provincia del Carchi, el sector de San Jerónimo $\left(00 \circ 40^{\prime} \mathrm{N}, \quad 77 \circ 53^{\prime} \mathrm{W}\right)$ en la zona de amortiguamiento de la Reserva Ecológica El Ángel. Esta reserva privada de propiedad de la Corporación Grupo Randi Randi se encuentra a $3200 \mathrm{msnm}$ en la Cordillera Occidental de los Andes. La vegetación en 
esta zona es un mosaico de formaciones vegetales: páramo de frailejones, humedales y bosque siempre verde montano alto (Figura 1). Entre las especies dominantes del dosel del bosque se encuentran: Weinmannia sps., (Hedyosmum cumbalense H. Karst. y Miconia tinifolia Naudin, entre otras. Mientras el páramo esta dominado por frailejones (Espeletia pycnophylla Cuatrec) y gramíneas de los géneros Agrostis y Calamagrostis.

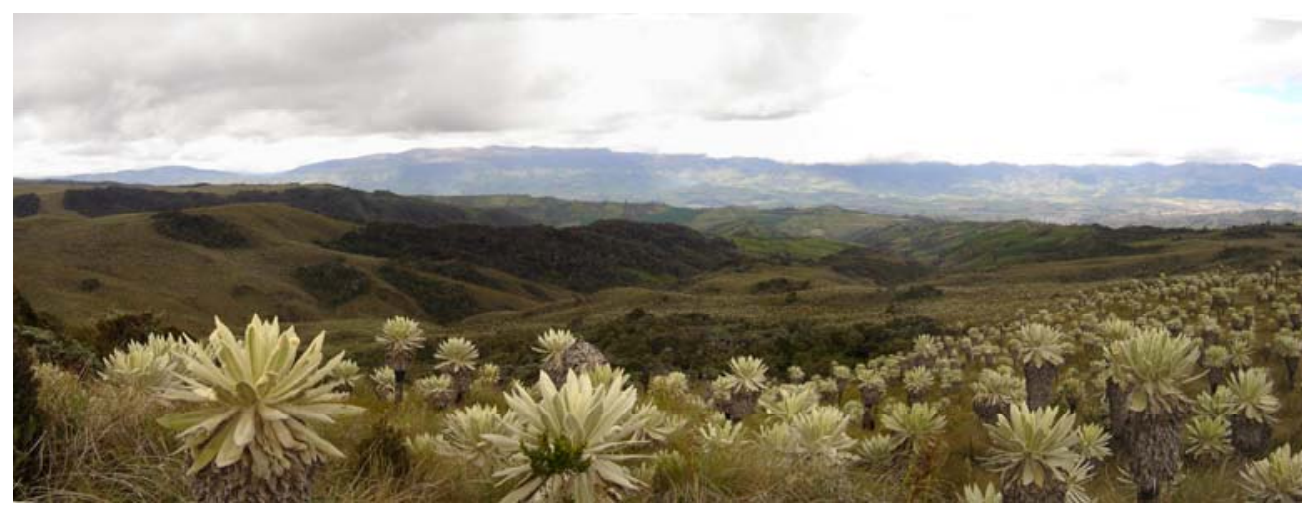

Figura 1. Fotografía de los remanentes de bosque siempre verde montano alto.

El corredor de hábitat tiene el objetivo de unir los fragmentos de bosque siempre verde montano que se encuentra dentro de la Estación Los Encinos. Para la formación de un corredor de hábitat, se trabajó a dos escalas: paisaje y parcela. A escala de paisaje se realizó un análisis multitemporal de fragmentación para definir un sitio idóneo para fomentar la conectividad; mientras que a escala de parcela se analizó el crecimiento de las especies plantadas para estructurar el corredor. A continuación se detalla cada análisis.

Análisis multitemporal de fragmentación

La fragmentación del bosque siempre verde montano alto de la Estación Los Encinos se analizó en escala cartográfica 1:60.000 con ayuda del software ArcView 3.2 y ortofotos del 17 de Febrero de 1966 y 14 de Noviembre de 1999, teniendo entre las dos una diferencia de 33 años. Para el trabajo se utilizó fotografías aéreas por dos razones: la primera porque son más baratas que una imagen satelital y la segunda es porque la diferencia de cambios sucesionales en bosques tropicales es más fácil distinguir en fotografías aéreas que en imágenes de satélite (Chinea, 2002).

Cada fragmento de bosque dentro de la Estación Los Encinos fue delimitado y diferenciado, obteniendo la superficie de cada uno; con estos datos se calculó un índice de fragmentación (IF) para cada año. Este IF corresponde a la relación entre el perímetro (P) y el área del bosque (A). Mientras mayor sea el perímetro - longitud de borde- sobre el área, habrá mayor fragmentación ( IF $=\mathrm{P} / \mathrm{A})$. El índice permite identificar: los cambios que se produjeron en los remanentes de bosque, durante este período de tiempo y los lugares que son más propensos a fragmentarse. Con esta información se puede priorizar los sitios donde es necesario realizar actividades de restauración.

Formación del corredor de hábitat

En base a los resultados de la fragmentación de bosque se seleccionó un área para formar un corredor de hábitat. Los dos remanentes de bosque estaban aislados $30 \mathrm{~m}$ entre cada uno, por la formación vegetal de páramo de frailejones desde hace más de 40 años. Al remanente más grande se consideró como ecosistema donador, mientras que al fragmento más pequeño fue el ecosistema receptor (Galindo-Leal, 1996). El corredor de hábitat se conformó con especies características de la formación vegetal de bosque siempre verde montano alto del norte del Ecuador. Al escoger las especies se tuvo en cuenta los límites ecológicos de distribución, determinados en el Catálogo de Plantas Vasculares del Ecuador (Jørgensen \& León-Yánez, 1999). La mayoría de plantas seleccionadas para el ensayo, han sido registradas por otras personas en remanentes de bosque siempre verde montano (Ulloa \& Jørgensen, 1995; Jørgensen et al., 1995; Cuamacás \& Tipaz, 1995). A pesar que el género Weinmannia, es el más abundante en los remanentes de la Estación Los Encinos, fue muy difícil conseguir plántulas de estas especies, por este motivo no se pudo probar con esa especie. Las plantas utilizadas en la conformación del corredor fueron: Buddleja sp. (quishuar), Macleania stricta A.C. Sm (chaquililu), Myrcianthes rhopaloides (Kunth) McVaugh (arrayán) Baccharis sp. (chilca), Aegiphila ferruginea Hayek \& Spruce (balso), Oreopanax ecuadorensis Seem (pumamaqui). Para formar el corredor de hábitat se utilizaron tres estrategias de disposición de las plantas:

a. Borde de bosque (BB). Las especies se plantaron en el borde del remanente de bosque más grande (entre 0.5 y 3 metros de la línea del bosque).

b. Reconformación de fragmento $(R F)$. En esta zona se procuró plantar en los claros y en los bordes del fragmento aislado hace más de 40 años, tratando de reestructurar su forma.

c. Unión de Fragmentos (UF). En este sitio se plantó las especies procurando reconformar la masa del 
bosque, además se retiró toda la paja y frailejones existentes de la zona con un azadón. Solo quedaron en pie las especies arbustivas y algunas plántulas de árboles.

En el caso de las dos primeras estrategias se aprovechó la sombra y el efecto borde, tanto del remanente grande como del fragmento pequeño. En la tercera quería evitar que la vegetación de páramo compita con las especies sembradas (Figura 2). La especies se plantaron a $1.50 \mathrm{~m}$ de separación.
Suelos y Aguas del Servicio Ecuatoriano de Sanidad Agropecuaria (SESA) del Ministerio de Agricultura y Ganadería de Ecuador. Es inevitable mencionar que es muy difícil adicionar compuestos al suelo cuando se planta varias especies de árboles o arbustos nativos, ya que cada una de ellos tiene requerimientos especiales de nutrientes del suelo. Para sobrellevar este problema es necesario que las características del suelo donde se va a plantar, sean similares a las características del suelo de un bosque nativo.

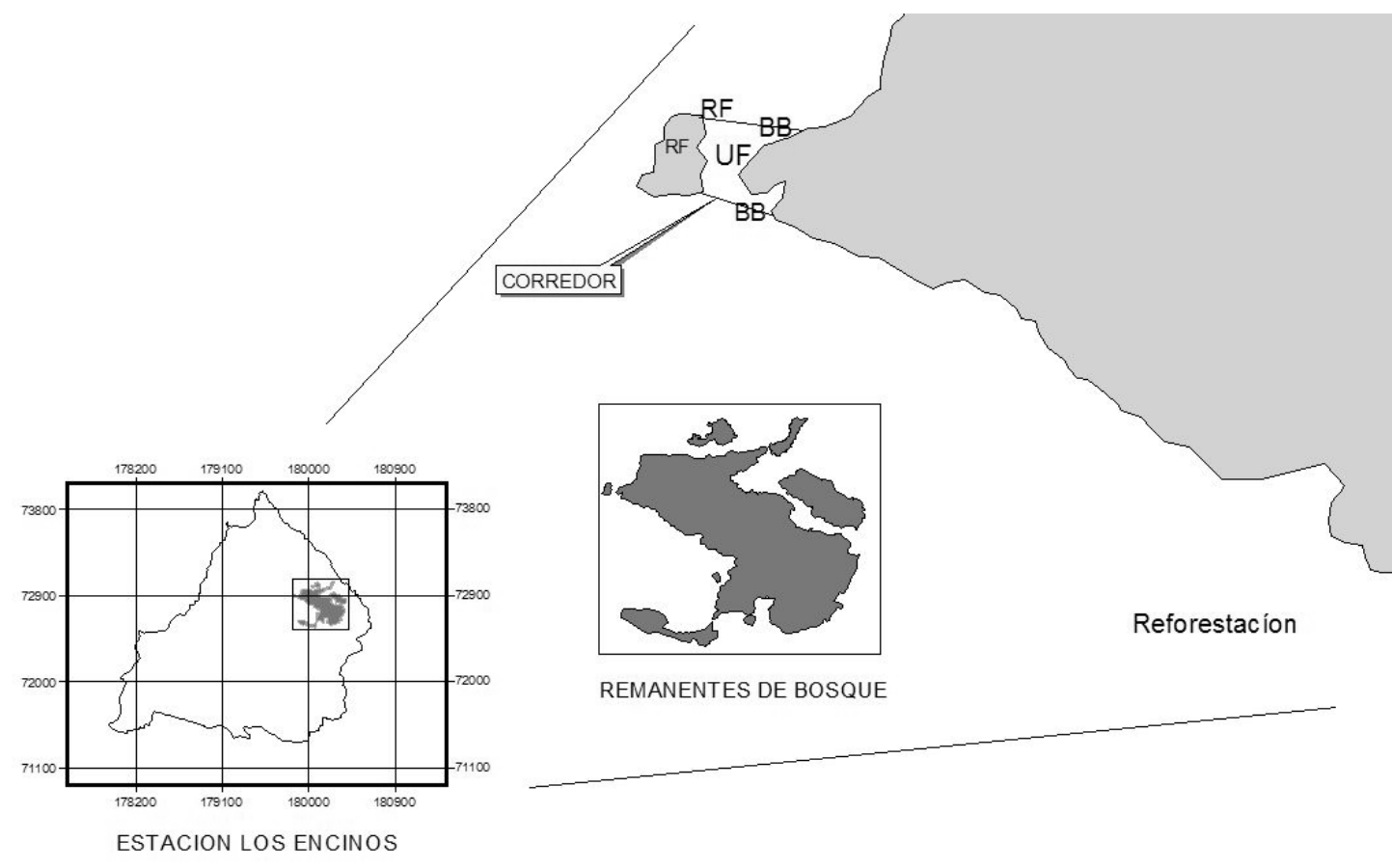

Figura 2. Ubicación del corredor de hábitat y de los diferentes ensayos.

Para tener un testigo con el cual comparar el crecimiento de las plantas de las tres estrategias para la formación del corredor, alejado de la matriz de bosque se plantaron las mismas especies de plantas en línea (tipo plantación). En este lugar no se retiró la vegetación del páramo de frailejones, solo abrió el espacio para poner las plantas. Esta parcela se denominó "Reforestación" (Figura 2), porque muchas organizaciones realizan programas de reforestación en el Ecuador siguiendo este parámetro (Galloway, 1986). El seguimiento de las plantas sembradas se realizó un mes después de la plantación y a los seis meses de ésta, el monitoreo del desarrollo de las plantas se efectuó al año y a los dos años de plantadas. El ensayo esta montado en la estación los Encinos para futuros monitoreos.

Las muestras tomadas para el análisis del suelo (a $0.30 \mathrm{~m}$ de profundidad) en los lugares en donde se efectuó los ensayos, fueron enviadas al laboratorio de

\section{Resultados y discusión}

Análisis multitemporal de fragmentación

En un período de 33 años (1966 a 1999), el remanente de bosque de Ceja Andina de la Estación Los Encinos ha cambiado su tamaño (Tabla 1). Para 1966, el área cubierta por bosque siempre verde montano alto era de $117836.855 \mathrm{~m}^{2}$ (11.78 ha), mientras que para el año 1999, el área cubierta por la misma formación vegetal era de $113417.755 \mathrm{~m}^{2}$ (11.34 ha). En estos 33 años se ha perdido, aproximadamente $4419.1 \mathrm{~m}^{2}(0.44 \mathrm{ha})^{*}$, por procesos naturales del sistema ecológico.

La pérdida de media hectárea en 33 años parecería un valor muy insignificante, pero al realizar un análisis de fragmentación (relación perímetro/área), se demuestra que aumenta el índice de fragmentación de

* Matemáticamente, el valor por 33 años, equivaldría a $133.91 \mathrm{~m}^{2}(0.013 \mathrm{ha})$ por año, esto dato ecológicamente es muy cuestionable, y arbitrario, porque la naturaleza no se comporta de la misma forma todo los años (fenómenos climáticos de El Niño y La Niña entre otros). 
0.787 del año 1966 a 1.133 para 1999 (Tabla 1). Este aumento de la fragmentación se evidenció por: la división de un fragmento de bosque en dos (6a y 6b) para 1999, la disminución del área de bosque y el aumento del perímetro (Tabla 1, Figura 3). Es necesario mencionar que en algunos de los espacios separados hace más de 33 años, no se encontró indicios de regeneración de bosque, más bien aumento el aislamiento y la paramización que es el ingreso del vegetación de páramo en los sitios más frágiles de los remanentes del bosque alto andino, en el ecotono línea de bosque- entre el límite del páramo y del bosque montano alto (Sarmiento, 2002). analizado, el número cuatro aumentó de tamaño en los 33 años (Tabla 1, Figura 3). Este aumento del área cubierta por vegetación natural, podría deberse a una regeneración natural que se suscitó en este fragmento, gracias a que la intensidad de la presión a la que fue expuesto no fue tan drástica como en los demás, o este fragmento por ser más grande fue más resiliente (En la fotografía aérea el bosque en esta sector parece mas denso). Es necesario acotar que conforme aumenta la intensidad con que se ha utilizado un sitio (Guariguata \& Ostertag, 2002), disminuye también la posibilidad de que se recupere por tal razón según Guariguata \& Ostertag (2002) "si la degradación de un sitio no es

Tabla 1. Análisis multitemporal de un remante de bosque de Ceja Andina

\begin{tabular}{|c|c|c|c|c|c|c|c|}
\hline & FEBRERO & 1966 & & NOVIEMBRE & 1999 & & Diferencia \\
\hline Fragmento & $\begin{array}{l}\text { Área } \\
\left(\mathrm{m}^{2}\right)\end{array}$ & $\begin{array}{l}\text { Perímetro } \\
\text { (m) }\end{array}$ & $\mathrm{IF}=\mathrm{PA}$ & $\begin{array}{l}\text { Área } \\
\left(\mathrm{m}^{2}\right)\end{array}$ & $\begin{array}{l}\text { Perímetro } \\
\text { (m) }\end{array}$ & $\mathrm{IF}=\mathrm{PA}$ & $\begin{array}{c}1966-1999 \\
\left(\mathrm{~m}^{2}\right)\end{array}$ \\
\hline 1 & 11311.07 & 512.93 & 0.045 & 10975.91 & 521.37 & 0.048 & 335.162 \\
\hline 2 & 2301.94 & 304.87 & 0.132 & 2011.58 & 289.972 & 0.144 & 290.355 \\
\hline 3 & 455.525 & 83.22 & 0.183 & 344.676 & 81.695 & 0.237 & 110.849 \\
\hline 4 & 1711.34 & 160.89 & 0.094 & 2733.07 & 371.165 & 0.136 & -1021.73 \\
\hline 5 & 6560.68 & 546.46 & 0.083 & 5902.03 & 537.289 & 0.091 & 658.65 \\
\hline $6(6 a) *$ & 95214.72 & 2014.30 & 0.021 & 90843.43 & 2035.69 & 0.022 & 4371.29 \\
\hline 7 & 281.582 & 64.35 & 0.229 & 220.247 & 58.201 & 0.264 & 61.335 \\
\hline $8(6 b-99)$ & & & & 386.814 & 73898 & 0.191 & -386.814 \\
\hline Total & 117836.86 & & 0.787 & 113417.76 & & 1.133 & 4419.10 \\
\hline
\end{tabular}

IF: índice de fragmentación

* Para mostrar la separación de los fragmentos del original se utilizará el numero seguido de un letra en orden ascendente a, b, c,...etc.

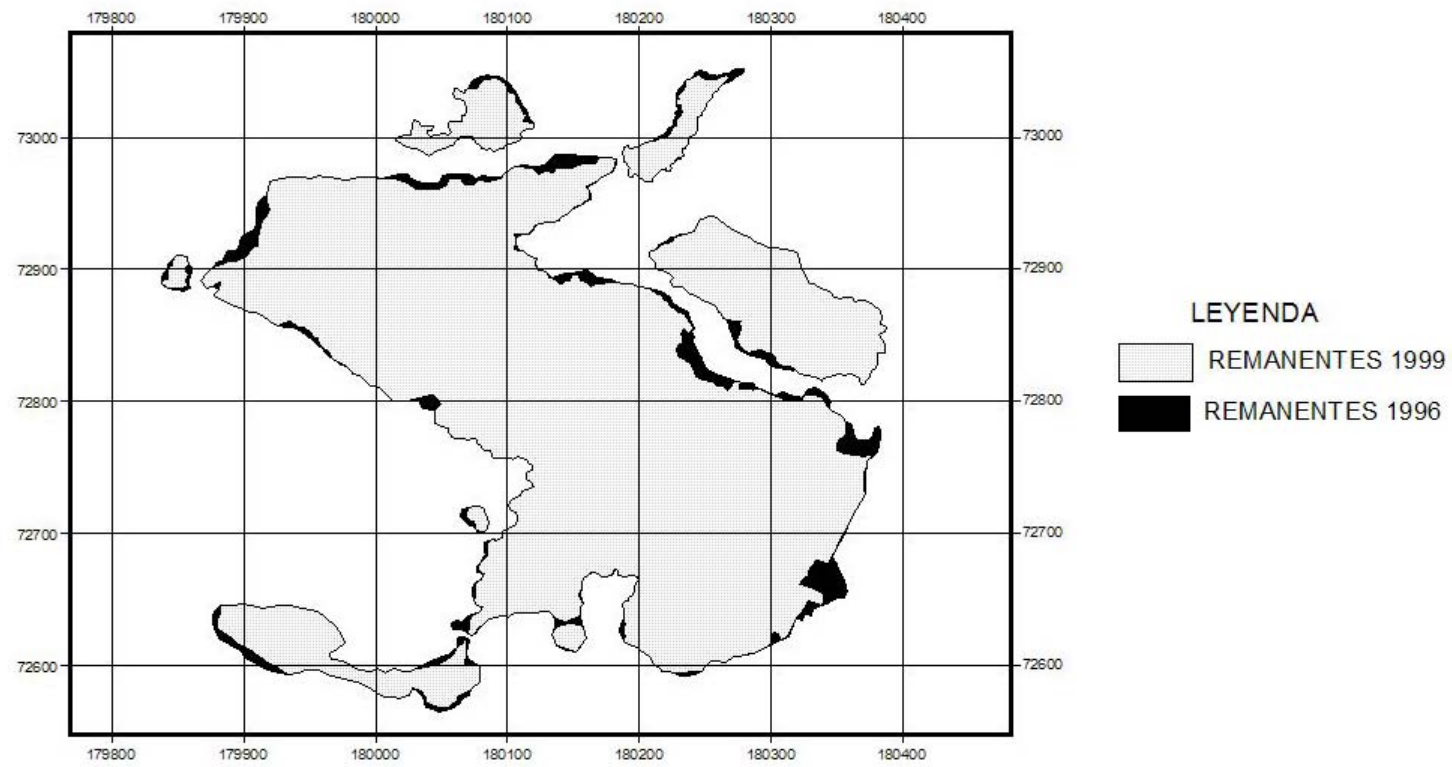

Figura 3. Sobreposición de los remanentes de bosque de los años 1996 y 1999 . particularmente aguda y si las fuentes de semillas (fragmentos más grandes, o

En contraposición a la disminución del tamaño de la mayoría de fragmentos del remanente de bosque ecosistemas donantes) se encuentran próximas, la sucesión natural podría ser una opción más barata y 
más viable para restaurar un bosque". Este resultado permite sugerir para futuras investigaciones, una nueva hipótesis: Los remanentes de bosque de ceja andina son un sistema dinámico, cuya recuperación natural (resiliencia) depende de la intensidad de la presión a la que son expuestos y de la funcionalidad del remanente.

Formación del corredor de hábitat.

\section{Características del Suelo.}

Los resultados de los análisis de suelo en las diferentes parcelas (Tabla 2) son muy similares entre sí, salvo por los contenidos de Manganeso (Mn). El Mn dentro del bosque y en suelo de la parcela de restauración, está evaluado como Bajo, mientras que en la parcela de "reforestación" es Medio. Es preciso aclarar que este compuesto es necesario para la formación de clorofila y que en la mayoría de suelos se encuentra en la cantidad suficiente y raramente debe ser añadido (Bruni, 1964). El Mn junto a otros microelementos son esenciales para el metabolismo de las plantas, pero por si solos no, ya que estos elementos son absorbidos en dosis mínimas (Guimarăes, 1963). En base a lo mencionado no fue necesario adicionar abonos, ni elementos correctivos al suelo.
Unión de Fragmentos (UF) $=3.4 \mathrm{~cm}$. El crecimiento entre estas tres parcelas y las plantadas alejadas del remante (reforestación) se encontró que hay una diferencia significativa (ANOVA $\mathrm{F}=3.331 \quad \mathrm{P}<0.05$ ). De estas tres zonas del corredor de hábitat, las plantas colocadas en la estrategia RF tuvieron el mejor crecimiento $(\mathrm{Z}=2.79961, \mathrm{P}<0.05)$ que las puestas como plantación (reforestación). Esto muestra que plantando cerca de los remanentes de bosque andino, en sus bordes (ampliando el fragmento) es posible ampliar la masa boscosa y quizás recuperar los remanentes de bosque andino.

\section{Análisis del crecimiento por especies}

Entre las especies plantadas en los ensayos se encontraba una especie endémica para el Ecuador, Oreopanax ecuadorensis Seem o pumamaqui. Esta especie según Montúfar (2000) "es común en los remanentes de vegetación andina, en cercas vivas y vegetación arbustiva a lo largo de los ríos". Esta planta está evaluada como especie de preocupación menor (LC), en el Libro Rojo de las Plantas Endémicas del Ecuador (Valencia et al., 2000). Esta especie tuvo un crecimiento en promedio de $\mathrm{BB}=8.3$ $\mathrm{cm}, \mathrm{RF}=6.8 \mathrm{~cm}, \mathrm{UF}=3.4 \mathrm{~cm}$, mientras que los individuos colocados lejos del remanente de bosque (reforestación) tuvieron un promedio de crecimiento

Tabla 2. Características del suelo de los lugares de los ensayos

\begin{tabular}{|c|c|c|c|c|c|c|c|c|c|c|c|c|}
\hline Sitio & $\mathbf{P h}$ & $\underset{\%}{\text { M.O. }}$ & $\begin{array}{c}\text { N.Total } \\
\%\end{array}$ & $\begin{array}{c}\mathbf{P} \\
\mathbf{p p m}\end{array}$ & $\begin{array}{c}\mathrm{K} \\
\mathbf{p p m}\end{array}$ & $\begin{array}{c}\text { Ca } \\
\text { ppm }\end{array}$ & $\begin{array}{c}\text { Mg } \\
\text { ppm }\end{array}$ & $\begin{array}{c}\text { Fe } \\
\text { ppm }\end{array}$ & $\begin{array}{c}\text { Mn } \\
\text { ppm }\end{array}$ & $\begin{array}{c}\mathrm{Cu} \\
\text { Ppm }\end{array}$ & $\begin{array}{c}\mathrm{Zn} \\
\text { ppm }\end{array}$ & $\begin{array}{c}\text { Clase } \\
\text { Textural }\end{array}$ \\
\hline \multirow[t]{2}{*}{ Bosque } & 4.96 & 32.94 & 1.65 & 0.50 & 30 & 86 & 9 & 13 & 1 & 0.32 & 0.20 & Orgánico \\
\hline & Ácido & A & A & B & B & B & B & B & B & B & B & \\
\hline \multirow[t]{2}{*}{ Corredor } & 4.93 & 33.76 & 1.69 & 4 & 70 & 40 & 7 & 16 & 3 & 0.45 & 0.14 & Orgánico \\
\hline & Ácido & A & A & B & $\mathrm{B}$ & B & B & B & B & B & B & \\
\hline \multirow[t]{2}{*}{ "Reforestación" } & 5.36 & 23.46 & 1.17 & 2 & 70 & 76 & 12 & 3 & 11 & 0.42 & 0.20 & Orgánico \\
\hline & Ácido & A & A & B & B & B & B & B & M & B & B & \\
\hline
\end{tabular}

A: Alto, M: Medio, B: Bajo, M.O: Materia orgánica, N.Total: Nitrógeno total

\section{Análisis del crecimiento de plantas por estrategias}

Las plantas colocadas para formar el corredor de hábitat tuvieron un crecimiento en promedio de 4.53 $\mathrm{cm}$ en seis meses, mayor que las plantadas lejos de los remanente de bosque en el ensayo denominado "reforestación", que crecieron en promedio de 3.94 $\mathrm{cm}$. Auque existe una diferencia en el crecimiento de las plantas, no existe una diferencia significativa (ANOVA $\mathrm{F}=0.4453, \mathrm{P}=0.5052 ; \mathrm{y} \mathrm{Z}=0.8619, \mathrm{P}$ $=0.1711$ ). Esto muestra que la colocación de plantas tratando de formar un corredor, como la ubicación de plantas alejada de los remanentes de bosque como plantación, son alternativas para repoblar de bosques la zona andina del norte del Ecuador. Pero con la primera estrategia se puede recuperar la funcionalidad de los remanentes, mientras que con la otra quizás no.

Las especies plantadas en las diferentes partes del corredor de hábitat crecieron en seis meses en promedio: Borde de Bosque (BB) $=5.2 \mathrm{~cm}$. Reconformación de Fragmento $(\mathrm{RF})=6.8 \mathrm{~cm} \quad y$ de $7.1 \mathrm{~cm}$ (ANOVA $\mathrm{F}=6.663, \mathrm{P}<0.05)$. A pesar que si existió una diferencia significativa en el crecimiento de las plantas en los diferentes sectores, ninguno de los sitios tuvo un desarrollo de las plantas más prominente que los otros.

El poco crecimiento que tuvo el Oreopanax ecuadorensis Seem en la estrategia del UF a diferencia de las otras dos estrategias para formación de un corredor de hábitat, se debe a que esta especie según Lojan (1992) "se puede desarrollar muy bien en semisombra", lo cual esta estrategia no producía. Esto muestra que cada especie tiene necesidades diferentes y que las estrategias para recuperar el bosque andino debería considerarlas. Además da la pauta para que en futuros programa de recuperación de bosques andinos, si se piensa en recuperar el acervo genético y la diversidad florística y no solo la masa boscosa, es necesario planificar programas de restauración, en los que se incluya una primera fase de plantación de especies nodrizas (arbusto o árboles de crecimiento 
rápido) que produzcan sombra a otras plantas, para que en una segunda fase se introduzcan especies importantes del ecosistema; de esta forma se esta replicando la dinámica sucesional de los bosques. Al contrario de lo que están haciendo ahora, algunas instituciones de plantar todas las especies en una sola campaña.

Es interesante notar que la Baccharis sp. (chilca) que es una especie arbustiva de la zona andina del Ecuador, tuvo un excelente crecimiento en promedio en la parcela UF $(X=13 \mathrm{~cm})$. Por lo que se sugiere que en futuras investigaciones de unión de fragmentos y restauración de bosque, se pueda utilizar a esta especie como nodriza. La ventaja de la "chilca" es el crecimiento rápido, por esta razón al poco tiempo de plantada, esta podría dar sombra a otras con crecimiento más lento, o que sean posteriormente plantadas. Así las otras plantas crecerían protegidas por esta especie y con el tiempo, ellas sobrepasarían el tamaño de estas nodrizas, pero ya robustecidas.

La especie como la Macleania stricta A.C. Sm (chaquilulu), no tuvo un buen desarrollo, incluso algunos individuos murieron en la estrategia BB. Esta situación debe ser evaluada en futuras investigaciones, ya que quizás es una especie que requiera: más sombra, otro tipo de suelo ${ }^{\dagger}$, o no resista quizás las características eco-fisiológicas del borde. Antes de seguir usando esta especie en recuperación de vegetación andina, es necesario investigar más a fondo sus requerimientos.

El arrayán (Myrcianthes rhopaloides (Kunth) McVaugh) creció mejor en la estrategia de Borde de Bosque $(X=3.1 \mathrm{~cm})$ porque este especie según Lojan (1992) "crece mejor en lugares húmedos". A parte de brindar humedad el borde protege a las plantas de la radiación y temperatura especiales de este ecotono entre el bosque andino y el páramo. El arrayán podría ser una especie importante en la ampliación de la masa de bosque de andino, si se la planta en los bordes de los fragmentos.

La especie Buddleja sp. (quishuar) creció mejor alejada de los remanentes de bosque $(X=5.2 \mathrm{~cm})$ en el ensayo denominado "reforestación", esta especie creció poco en las estrategias de BB $(X=0.5 \mathrm{~cm})$ y UF $(X=2.5 \mathrm{~cm})$. Este género según Lojan (1992) "se adapta muy bien a zonas abiertas, por esta razón se la utiliza para cerca vivas". Por el mejor crecimiento que tuvo esta especie, en el ensayo alejado del bosque se la podría utilizar par recuperar la masa de bosque en lugares donde se ha perdido totalmente.

Monitores anuales del desarrollo de las plantas

† Por ejemplo esta especie como otras de la familia Ericaceae se caracteriza por adaptarse a suelos ácidos, por la presencia de micorrizas en las raíces. La falta de estos hongos, o en condiciones donde ellos no sobreviven, las plantas no se desarrollan bien.
En el mes de Noviembre del 2005 en Ecuador hubo una helada que ocasionó pérdidas económicas fuertes a las personas agricultoras, además de la muerte de varios individuos plantados en algunos programas de reforestación de la provincia del Carchi al norte del país. En la parcelas de la presente investigación, las plantas sembradas alejadas del bosque (reforestación) tuvieron mayor índice de mortandad que las que conformaban el corredor de hábitat. Entre las diferentes estrategias para conformar el corredor, las plantadas en el borde del bosque (BB) y reconformación de fragmento $(\mathrm{RF})$, su índice de no fue importante, mientras que la plantadas en la estrategia UF, fue mayor que las otras dos estrategias, pero menor que el de la parcela de "reforestación" que solo sobrevivieron 26 plantas.

Durante un monitoreo realizado en Noviembre del 2006 se encontró que las especies plantadas alejadas del bosque, la mayoría habían muerto por la continuas heladas y la competencia con las especies de páramo $\mathrm{y}$ otros factores. Mientras que las plantas de las estrategias RF y BB se mantenían vivas y creciendo, salvo las que murieron en la helada de Noviembre del 2005. Algunos individuos están presentando enfermedades (hongos) que también están atacando a las hojas de otras especies del bosque, estos patógenos quizás son nativos de este ecosistema, porque se los encontró en otros parches de Ceja Andina del remanente objeto de estudio. Los hongos atacaron a las hojas de arrayán (Myrcianthes rhopaloides (Kunth) McVaugh) pero ellas siguen creciendo, parece que se están adaptando como el resto de especies del bosque a convivir con esta enfermedad. Es preciso investigar la influencia de estos hongos al desarrollo de estas plantas y las del bosque.

Las nuevas hojas del Oreopanax ecuadorensis Seem (pumamaqui) están siendo comidas al parecer por la guanta de monte (Agouti sp.), impidiendo el desarrollo normal de esta planta. En la estrategia de UF las plantas están compitiendo otra vez con las especies características del páramo de frailejones, que ha vuelto a repoblar la zona, pese a que se la saco antes de colocar las plantas el año 2004. Este hecho confirma lo propuesto por Sarmiento (2002) "sobre a paramización del bosque andino, que es el ingreso del páramo en los sitios más frágiles del remanente", por la fácil dispersión de las especies de las familias Asteraceae y Poaceae que dominan el páramo de frailejones.

\section{Conclusiones}

La recuperación de los bosques de Ceja Andina y la formación de corredores entre los fragmentos que actualmente existen es difícil. Hasta la fecha no se tiene suficiente información para hacerlo y gente tomadora de decisión, continúan con grandes campañas de reforestación, que están formando cualquier cosa menos bosque andino, en las cuales 
incluso en su planificación, no se esta pensando en los remanentes existentes. Después del análisis del seguimiento del crecimiento y el monitoreo del desarrollo de las plantas por dos años, se sugiere que la mejor estrategia para conformar un corredor de hábitat es plantar las especies en los bordes de los fragmentos bosques y paso a paso ir ampliando el área de estos remanentes. La restauración es un proceso que conforma una cadena de pasos a largo plazo, con muchas piezas, cada una de la cuales es necesario que sea investigada y evaluada, en beneficio de la conservación de la biodiversidad de los bosques andinos, cada día más escasa.

\section{Agradecimientos}

Esta investigación y las actividades que la respaldaron fueron realizadas gracias al soporte de proyecto Manejo colaborativo de recursos naturales en cuencas andinas del norte del Ecuador (MANRECUR III) financiado por el International Development Research Center y los monitoreos del crecimiento de plantas gracias al proyecto Conservación Comunitaria financiado por la MacArthur Foundation, ambos ejecutados por la Corporación Grupo Randi Randi. Agradezco a mis compañeros y compañeras de trabajo, a las amigas-os que me apoyaron en la fases de campo y de laboratorio, en especial a Susan V. Poats por su apoyo desde el inicio de la investigación, hasta la elaboración del presente documento. Además agradezco a las instituciones que apoyaron de alguna manera la presente investigación: Herbario Nacional del Ecuador (QCNE), Ministerio de Ambiente del Ecuador en El Ángel y la Escuela Politécnica del Ejército (ESPE). Igualmente agradezco los comentarios de Felipe Ríos y del otro revisor anónimo.

\section{Literatura citada}

Bennet A. 2003. Linkages in the landscape: the role of corridors and connectivity in wildlife conservation. IUCN. Gland, Switzerland.

Bruni G. 1964. Química Inorgánica. XII Edición. Editorial Hispanoamericana. España.

Cuamacás B. \& Tipaz G. 2005. Árboles de los Bosques Interandinos del Norte del Ecuador. Monografía No 4. Publicaciones del MECN. Quito.

Chinea J. 2002. Teledetección del Bosque. Páginas 625-646. En: Guariguata M \& G. Katan (Eds.) Ecología y Conservación de Bosques Neotropicales. Ediciones Libro Universitario Regional, Costa Rica.
Galindo-Leal C. 1996. Métodos Cuantitativos para el Manejo de Diversidad Biológica. Programa de Investigación Tropical. Centro para la Biología de la Conservación Universidad de Stanford.

Galloway G. 1986. Guía sobre la Repoblación Forestal en la Sierra Ecuatoriana. Proyecto DINAF-AID. Ministerio de Agricultura y Ganadería del Ecuador, Quito.

Guimarăes D. 1963. Súmula Geoquímica. Boletim Nos 115. Imprensa da Universidade de Minas Gerais, Rio de Janeiro.

Guariguata M. \& Ostertag R. 2002. Sucesión Secundaria. Páginas 591-623. En: Guariguata M. \& G. Katan (Eds.) Ecología y Conservación de Bosques Neotropicales. Ediciones Libro Universitario Regional, Costa Rica.

Jørgensen P.M., Ulloa C., Madsen J.E. \& Valencia R.1995. A floristic Analysis of the High Andes of Ecuador. Páginas: 221-237. In: Churchill S., Balslev H., Forero E. \& Luteyn H. (Eds.). Biodiversity and Conservation of Neotropical Montane Forest. New York Botanical Garden Press.

\& León-Yanéz S. (Eds.) 1999. Catalogue of The Vascular Plantas of Ecuador. Monographs in Systematic Botany. No 75.

Lojan L. 1992. El Verdor de los Andes. Árboles y arbustos nativos para el desarrollo forestal alto andino. Proyecto Desarrollo Forestal Participativo En Los Andes, Quito.

Luteyn J. 1999. Páramos. A checklist of plan diversity, geographical distribution and botanical literature. Memoirs of the New York Botanical Garden. Vol. 84.

Montúfar R. 2000. Araliaceae. Páginas 87 y 88. En: Valencia R., Pitman N., León-Yanéz S. \& Jørgensen P.M. (Eds.) Libro rojo de la plantas endémicas del Ecuador 2000. Publicaciones del Herbario QCA. Pontificia Universidad Católica del Ecuador. Quito.

Neill D. 1999. Vegetación del Ecuador. En: Jørgensen P. M \& S. León-Yanéz (Eds.). Catalogue of the Vascular Plantas of Ecuador. Monographs in Systematic Botany. Missouri Botanical Garden. No 75.

Sarmiento F. 2002. Impulsores de cambio del paisaje: Dinámica de las líneas de árboles en la Montología Neotropical. Ecotropicos. 15(2): 129-146

Ulloa C. \& Jørgensen P.M. 1995. Árboles y arbustos de los Andes del Ecuador. Segunda Edición Edt. Abya-Yala, Quito.

Valencia R., Cerón C., Palacios W. \& Sierra R. 1999. Formaciones Vegetales de la Sierra del Ecuador. En: Sierra R. (Ed.). 1999. Propuesta Preliminar de un Sistema de Clasificación de Vegetación para el Ecuador Continental. Proyecto INEFAN/GEF-BIRF y EcoCiencia. Quito, Ecuador

, Pitman N., León-Yanéz S. \& Jørgensen P.M. 2000 (Eds.). Libro rojo de las plantas endémicas del Ecuador 2000. Publicaciones del Herbario QCA. Pontificia Universidad Católica del Ecuador, Quito

${ }^{1}$ Corporación Grupo Randi Randi, Casilla17-11-6102. Quito-Ecuador, davsua2@yahoo.com 